\title{
Multilevel Pixel Colour Values Scrambling Based on Shifting Image Technique
}

\author{
Ahmed Bashir Abugharsa \\ Faculty of Information \\ Technology University of \\ Misurata, Misrata Libya.
}

\author{
Hamida Mohamed Almangush \\ Faculty of Information \\ Technology University of \\ Misurata, Misurata Libya
}

\begin{abstract}
Image scrambling is a useful approach to protect image data by confusing the image and thus prevent it from being misused. The issues concerning the relationship among the adjacent pixels of the scrambled image, the weakness of visual leakage, and similar histogram plots being produced, all affect the image scrambling process. This paper is aimed at determining the iteration level that is extracted from a secure key and a key mask for building a scrambling table which is used in the scrambling processes and modifying the pixel colour values. The technique begins by modified pixel colour values. These pixel colour values are then scrambled by reduce or the increase (modified) the pixel colour values in the image between the range of the colour (0 to 255) by using a vertical and horizontal scrambling method based on a scrambling table. The experimental results of the paper show that the pixel colour values scrambling technique has reduced the relationship among the image elements. This occurs because the entropy value of the scrambled images has increased and the correlation value is lower. Moreover, the scrambling degree of the scrambling technique has larger values for the scrambled image. Subsequently, the pixel colour value modification in the technique helps to confuse and amend the pixel colour values of the scrambled image to produce a different histogram plot when compared with plots made from the original images.
\end{abstract}

Keywords: Block Image Scrambling; Scrambled Image; Block Image Scramble; pixel scramble.

\section{INTRODUCTION}

The world is facing daily rapid changes in technology due to swift advances in computing, networks and communications. These advances have opened networks and individual machines to a wide range of abuse by offenders who abuse the technologies in many criminal activities. In other words, they use technologies in various Cybercrimes [1]-[27]. A study found that there are relatively few criminal and civil cases which do not apply digital facilitation. It has been estimated that over 85 percent of criminal and civil prosecution cases are committed through the use of digital technology [2]. The impact of the Internet has contributed to the demand for digital media such as audio, image, and video. It has not so much affected the creation of these media, but more on the reproduction and distribution, while in open networks most of the information is kept electronically. Therefore, the demand for protection of such images has gained more importance [3]-[28] The objective of image scrambling is to generate a non-intelligible image which prevents the human visual system or a computer vision system from understanding the true content. An authorised user is empowered to descramble the image using information regarding the scrambling method and the variables used in that processing in order to decipher the image. Image scrambling has been proposed as a way to mitigate such issues since way back in 1960 when the first documented system to do so emerged [4].

The approach at that time involved scrambling, concealing or encoding information and unscrambling and decoding the received images using line screens and grids consisting of opaque and transparent lines. Over the years, image scrambling has evolved into two streams. One is based on matrix transformation to shift coordinates and the other makes use of permutations of the pixel coordinates.

Most of the scrambling approaches are based on an Arnold Transform or a combination of the Arnold Transform with other techniques [5]-[8]. However, these methods are applicable only to equilateral images. If the images are not equilateral, meaning the width and height are the same, then they have to be padded with values to make them equilateral [9]. Since most of these techniques do not use a 'key' that provides additional protection, Zhou et al. [10] proposed a Fibonacci P-code based scrambling algorithm which requires two parameters to be known by the receiver side to descramble the images. Even though this is certainly a favourable development over the other methods, just two numbers do not provide adequate protection. Wang et al. [11] proposed an approach for optical image scrambling with a binary Fourier transform computer-generated hologram and pixel-scrambling. For this kind of encryption algorithm, the orders of the pixel scrambling and the encrypted image need to be used as the keys to decrypt the original image.

Other researchers [12]-[15] have attempted scrambling using random sequences based on chaos or pseudo random number generation based on parameters. Zhou et al. [16] proposed an algorithm using an M-sequence to shuffle image coordinates using two parameter keys. The M-sequence is a maximum length sequence that has been used in spread spectrum communications. It is a pseudo random noise sequence. In this approach, the authorised user is given the shift parameter $r$ and the distance parameter $\mathrm{p}$ which are used to generate a 2-D M-sequence to descramble the scrambled image. Ye [12] proposed a scrambling 
method based on a chaotic cellular automata, which is used to scramble the digital image as a pre-treatment for the watermarking process. El-Latif et al. [17] presented a method for the scrambling and descrambling of digital images. When a third person intercepts chaotic images, the parameters of the scrambling algorithm are secret. Image scrambling requires that the images after scrambling should have lower intelligibility. $\mathrm{Gu}$ and Han [18] presented an image scrambling algorithm based on chaotic sequences. The chaotic sequence is generated using three parameters and the algorithm typically has to be iterated 100 times to generate a non-linear sequence. This introduces high complexity and the resulting scrambled image histogram is modified in the process. Digital image scrambling cannot change the resolution of the images [19]. The images after scrambling show no difference or have no great difference from the original images, and can accurately express the content or meaning of the original images [12],[20]. Compared with traditional textual data, digital images have a greater data volume, meaning that digital images have greater plain image space and scrambled image space. It is most important that autocorrelation of digital images is directly expressed in the direction of orthogonality and various angles of inclination. As a one-dimensional signal sequence, text has no autocorrelation [21]. Zhou et al. [16] proposed to consider a scrambling algorithm which is used to influence the autocorrelation of images. This is because the worse the autocorrelation, the better the scrambling effect and the worse the intelligibility of the images after scrambling.

In this paper, a new technique is proposed for an image scrambling algorithm based on the pixel colour values of the image blocks. The technique uses blocks of an image by scrambling the pixel colour values of image blocks based on vertical and horizontal scrambling approaches depending on shifting technique.

\section{MATERIALS AND METHODS}

\subsection{The key mask}

The Key Mask is a one-dimension array containing a set of values and the array has the same length as the secure key. The values in the key mask array are extracted from the secure key by using the Key Code Table that is fixed in the algorithm. Figure 1 shows the steps involved in the key mask algorithm.

This algorithm is developed to generate the key mask by using the secure key and key code table.

\section{Algorithm Create_Key_Mask}

1: Input the secure key and load key code table

2 : Let length =get the secure key length

3: Let counter $i=1$

4: While (counter i <= length) do

4.1: item $=$ get the value of the position $($ counter $i)$ in the secure key;

4.2: Let counter $\mathrm{j}=1$;

4.3: While (counter $\mathrm{j}<=120$ ) do

If(item $=$ the value of the position (counter $j)$ in the key code table ) then

The value in position(counter i)of the Key mask = Key

code table[j].value;

End If

Increment counter $j$

End While

4.4 Increment counter i

End While

5: End Create_Key_Mask

6: Output: Key Mask
Fig. 1: Steps in the Algorithm for the Key Mask

\subsection{Iteration level}

The iteration level is an integer number derived from the key mask. Increasing the number of iterations during the image scrambling stage leads to greater scrambling without a leakage problem. However, the relationship between the level of iterations and scrambling depends on the relationship between the values in the key mask. The advantage of the level of iterations is used in the scrambling phase for a greater scrambling degree. Figure 2 shows the steps involved in the iteration level algorithm.

This algorithm is proposed to generate the iteration level by using the key mask.

Algorithm Calculate_Iteration_Level (key mask)

1: Load the key mask;

2: Let length $=$ get the key mask length, counter $i=1$;

3: While (counter $\mathrm{i}<=$ length) do

3.1: item $=$ get the value of the position (counter i)

in the key mask;

3.2: Let iteration $=0$;

3.3: While (item > 9) do

Let value $=$ item $\bmod 10$;

Let item $=$ item div 10

Let iteration $=$ iteration + value

End While

3.4 Let iteration $=$ iteration + item;

3.5 Increment counter i

End While

4: End Calculate_Iteration_Number

Fig. 2: Steps in the Iteration Level Algorithm

\subsection{Scrambling table}

The scrambling table is considered as a key to the whole process for the developed algorithms. It is the output of the mathematical function of which the factors are the key mask, block size and the dimensions of the original the image. The mathematical function generates a number value for all the cells of the scrambling table that are used by the algorithms to jumble the image pixel colour values to new values in the scrambled image. The key mask that is extracted from the secure key is used as an index to the columns of the scrambling table. The index of the columns in the original image is used as an index to the scrambling table in the vertical scrambling approach and the index of the rows in the original image is used as an index to the scrambling table in the horizontal scrambling approach. Figure 3 represents the algorithm to generate the scrambling table.

The combination of the mathematical function, the key mask, block size and the dimensions of the original image are used to build the scrambling table that is used to change the pixel colour values of the original image. The key mask and the mathematical function of this approach are used to play the main role in building the scrambling table, which is applied to generate the scrambled image with a different blocks size. The scrambling process refers to the operation of increase and decrease the pixel colour values of the image blocks. 


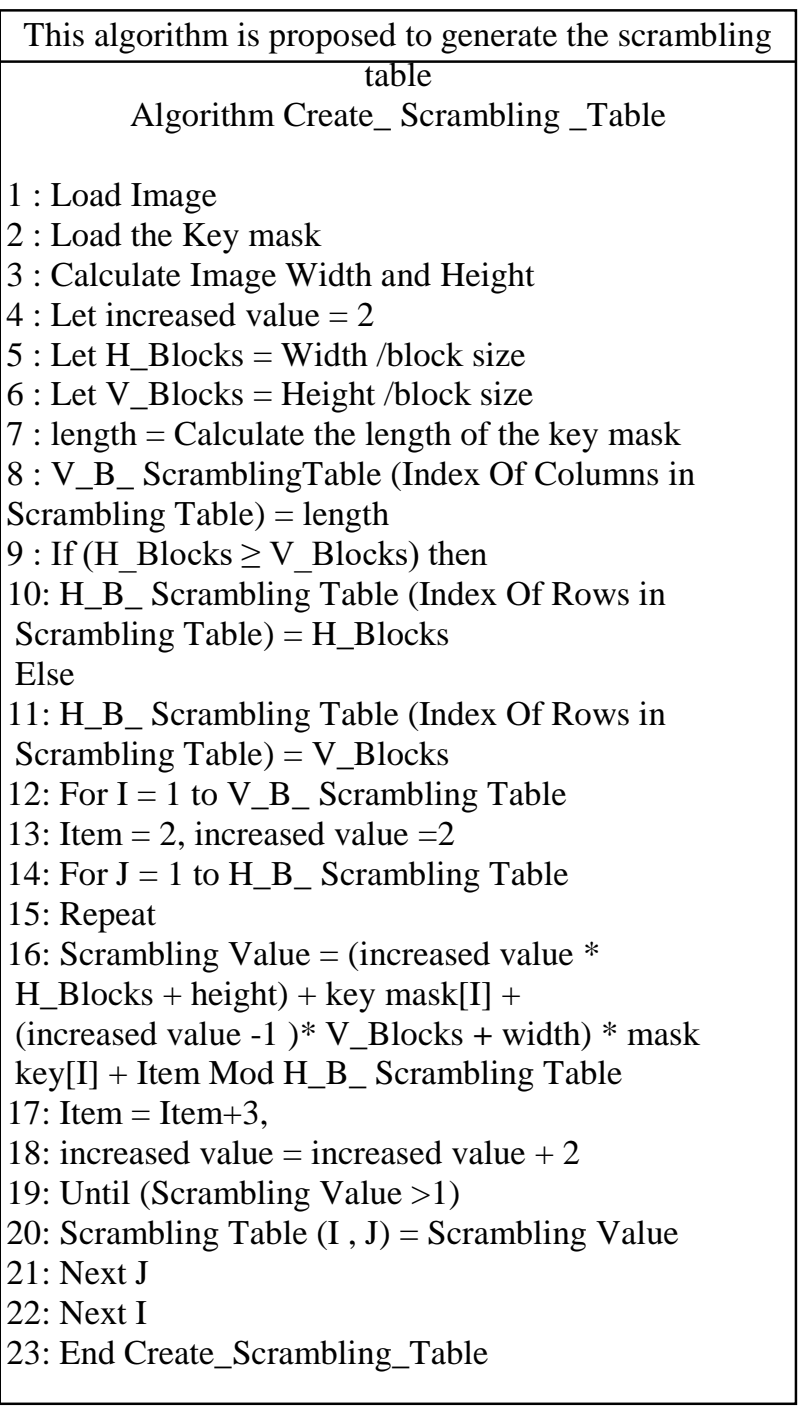

Fig. 3: Steps to generate the Scrambling Table

\subsection{Scrambling Pixel Colour Values}

The main step is changing or modified the pixel colour value (RGB) by using the scrambling pixel colour value algorithm based on shifting technique. This process is initiated by scrambling the pixel colour value which jumbles the colour between the boundaries of the image colours according to the values in the scrambling table by using the discussed formula to prevent the problem of underflow and overflow. In this method, the RGB value of a pixel is altered, the R value of the pixel is modified to another value according to the scrambling table values. In a similar manner, the $\mathrm{G}$ and $\mathrm{B}$ values of the pixel are also changed. These techniques are explained in the next section.
Change the pixel colour value by using the scrambling table.

Algorithm Scrambling Pixel_Colour_Values

Algorithm Scrambling Pixel_Colour_Value( Block,

ValueofScramb_Table )

1: Calculate Block Width and Height;

2: For $\mathrm{i}=1$ to Bl_height do

3: For $\mathrm{j}=1$ to $\mathrm{Bl} \_$width do

3.1 Block $[\mathrm{i}, \mathrm{j}] \cdot \operatorname{Red}=256+\mathrm{Block}[\mathrm{i}, \mathrm{j}] \cdot \operatorname{Red}+$

ValueofScramb_Table Mod 256

3.2 Block $[\mathrm{i}, \mathrm{j}] \cdot$ Green $=256+$ Block$[\mathrm{i}, \mathrm{j}] \cdot$ Green +

ValueofScramb_Table Mod 256

3.3 Block[i,j].Blue $=256+$ Block $[\mathrm{i}, \mathrm{j}] \cdot$ Blue +

ValueofScramb_Table Mod 256

4: Next j

5: Next i

6: End Scrambling Pixel_Colour_Value

7: Output: New Pixels Colour Value

Fig. 4: Steps to determine the New Pixel Colour Values

\subsection{Scrambling the colour value based on shifting technique}

The image scrambling technique involves vertical and horizontal movement approaches. The process is scrambling the pixel colour values. This is achieved by modifying (scrambling) the pixel colours between the boundaries of the image colours ( 0 to 255 ) according to the values in the scrambling table by using the discussed formula to prevent the problem of underflow and overflow. The main difference between these two approaches is how the pixel colour values are modified and how the pixel colour values are updated within the new scrambled image. In the vertical scrambling approach the pixel colour values are modified from bottom to top while in the horizontal scrambling approach the pixel colour values are modified from left to right [22]. An overview of Scrambling the colour value based on the shifting technique is shown in Figure 5.

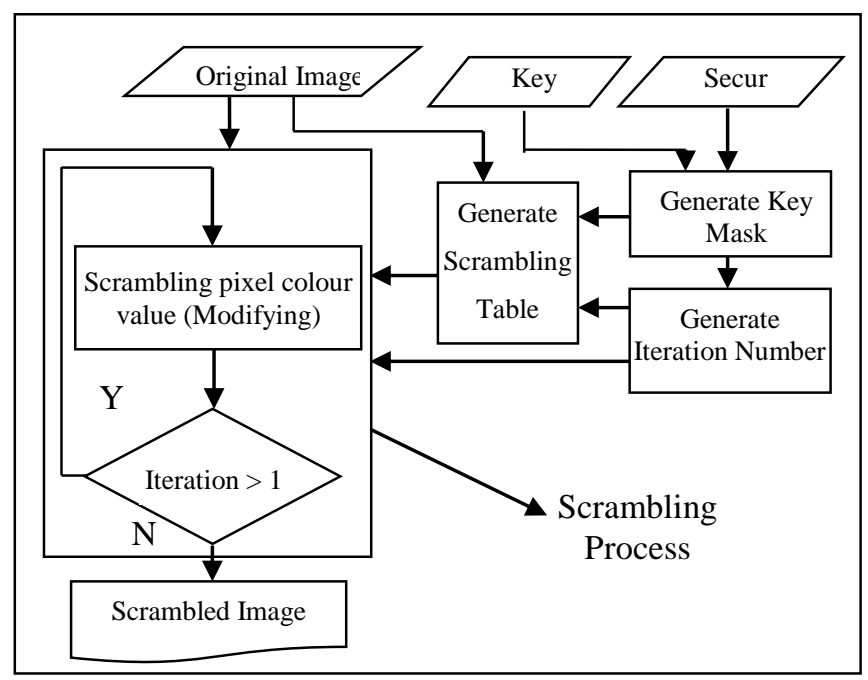

Fig. 5: Overview of the Scrambling the colour value based on the shifting technique 
The Scrambling the colour value based on the shifting technique works as follows:

1. Load the original image and secure key and then divide the original image into a different blocks size. Each block has the same number of pixels.

2. Generate the key code that will be used to extract the key mask from the secure key.

3. Call the key mask algorithm to create the mask key table. This step is very important in the proposed processes for the iteration level, scrambling table and scrambling.

4. Call the iteration level algorithm to generate the iteration level that can control the repetition of the scrambling processes.

5. Call the scrambling table algorithm to generate the scrambling table by combining the mathematical function, the width and height of original image and the different blocks size and the key mask to build the scrambling table that will be used to shift the rows and columns of the image. The key mask and the mathematical function of this approach are used to play the main role in building the scrambling table. The scrambling process refers to the operation of dividing and modified an arrangement of the original image.

6. The main idea of the scrambling process contains two steps, scrambling the pixel colour values horizontally and scrambling the pixel colour values vertically.

By calling the pixel colour value algorithm, the process of scrambling the pixel colour values takes place vertically and horizontally which modifies (scrambles) the pixel colour values between the boundaries of the image colour according to the values in scrambling table by using the proposed formula to prevent the problem of underflow and overflow. In this process, the RGB value of a pixel is changed to another value according to the scrambling table values.

7. The relationship among the elements of the image will be decreased and thus it becomes difficult to predict the value of any given pixel from the values of its neighbours. The clear information present in an image is due to the relationship among the image elements. The perceivable information can be reduced among the image pixels using Scrambling the colour value based on the shifting technique. Also, changing the pixel colour values can increase the level of security in the scrambled image by producing a new histogram which is different from the histogram of the original image. Thus, it becomes even more difficult to predict the value of any given pixel from the values of its neighbours.

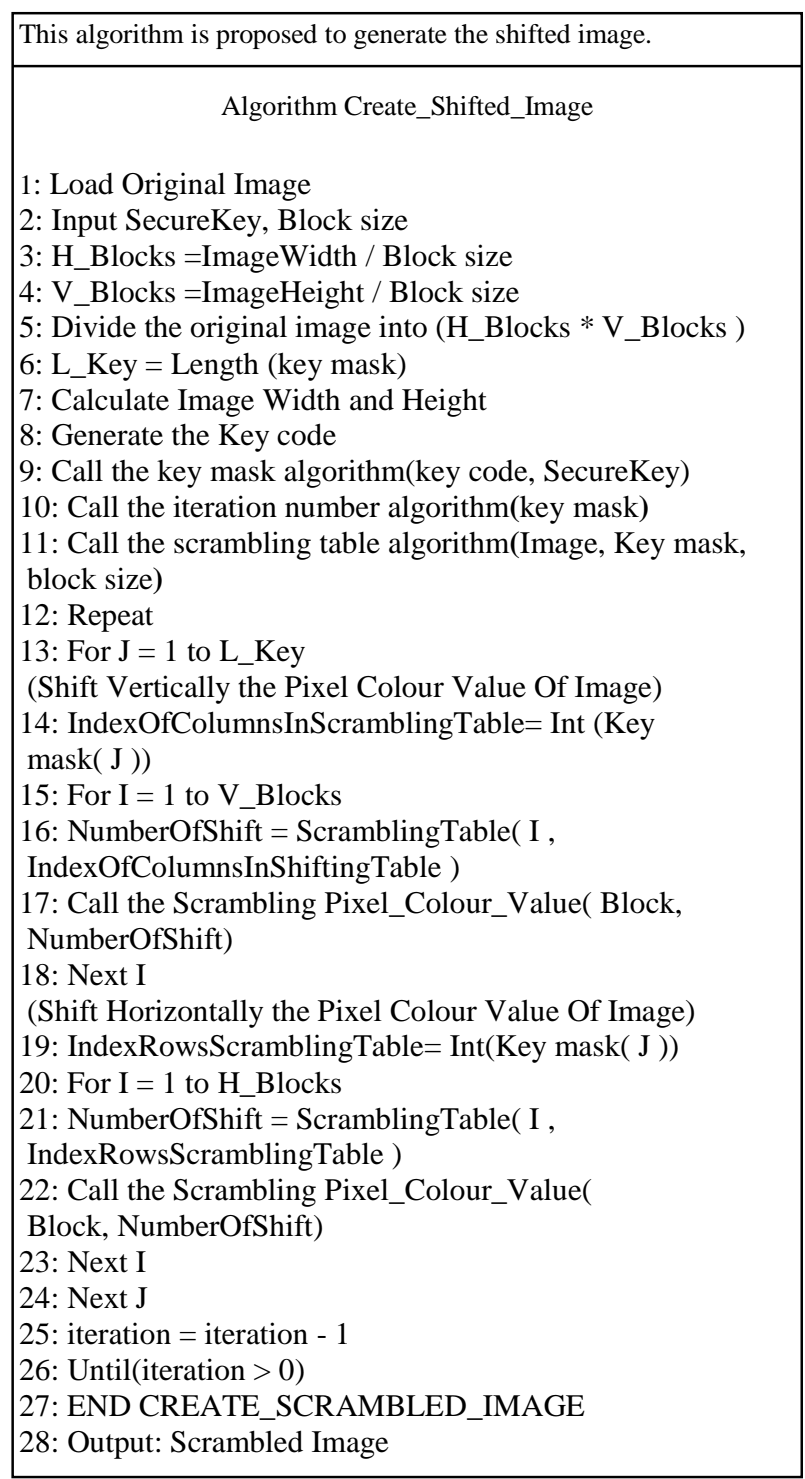

Fig. 6: Steps to generate the Scrambled Image

\section{EXPERIMENTAL DETAILS AND RESULTS}

A good quality scrambled algorithm should be strong against all types of attack, including statistical and brute force attacks. Some experiments are given in this section to demonstrate the efficiency of the proposed technique. The original image is first loaded by the Scrambling the colour value based on the shifting algorithm to separately build a new scrambled image respectively with different block sizes $(1$ pixel $\times 1$ pixel, 3 pixels $\times 3$ pixels, 10 pixels $\times 10$ pixels) using the same iteration level (40 levels). Therefore, three different scrambled images are obtained. The different blocks size and the block sizes in each case are shown in Table 1. 
Table 1. Different cases of number of blocks

\begin{tabular}{cc}
\hline \hline Case number & Block size \\
\hline 1 & 10 Pixels * 10 Pixels \\
2 & 3 Pixels * 3 Pixels \\
3 & 1 Pixel * 1 Pixel \\
\hline \hline
\end{tabular}

In this paper, original images are selected as shown in Figure 7 for use in this experiment.

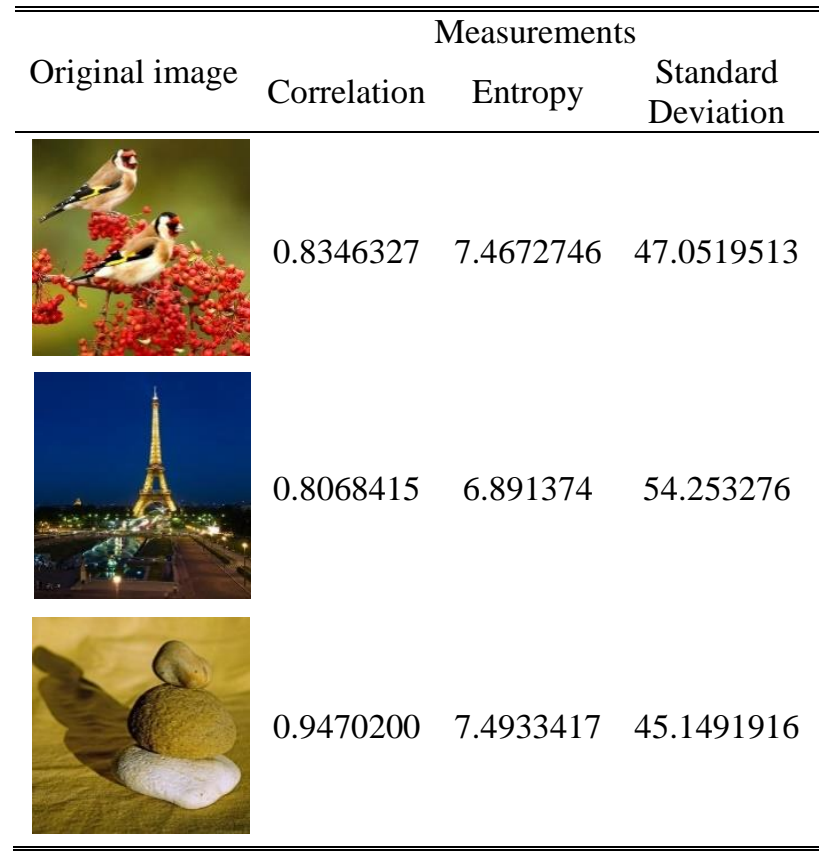

Fig. 7: the original images used in the experiment

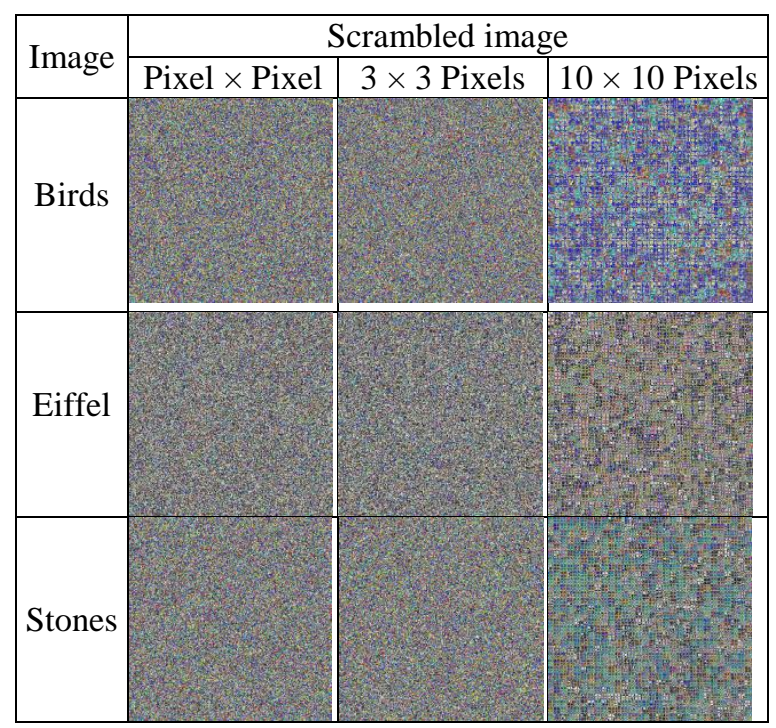

Fig. 8: Result of Scrambled image for all the cases by using a 40 Iteration level

\subsection{Correlation of two adjacent pixels}

A correlation is a statistical measure of security that expresses a degree of relationship between two adjacent pixels in an image or a degree of association between two adjacent pixels in an image. The aim of correlation measures is to keep the amount of redundant information available in the encrypted image as low as possible [23]. Equation (1) is used to study the correlation between two adjacent pixels in the horizontal, vertical, diagonal and anti-diagonal orientations [24].

$C_{r}=\frac{N \sum_{j=1}^{N}\left(X_{j} \times Y_{j}\right)-\sum_{j=1}^{N} X_{j} \times \sum_{j=1}^{N} Y_{j}}{\sqrt{\left(N \sum_{j=1}^{N} X_{j}^{2}-\left(\sum_{j=1}^{N} X_{j}\right)^{2}\right)} \times\left(N \sum_{j=1}^{N} Y_{j}^{2}-\left(\sum_{j=1}^{N} Y_{j}\right)^{2}\right)}(1)$

where $x$ and $y$ are the intensity values of two neighbouring pixels in the image and $N$ is the number of adjacent pixels selected from the image to calculate the correlation.

\subsubsection{Analysis of the relationship between the pixel colour value of number Blocks and the Correlation of Scrambled image}

Table 2 and Figure 9 summarise the correlation results of the scrambled image for all cases by using a different blocks size with 40 iteration levels.

Table 2: Correlation value results of the Scrambled image for all cases

\begin{tabular}{ccc}
\hline Case & Number of blocks & $\begin{array}{c}\text { Correlation of Scrambled } \\
\text { image }\end{array}$ \\
\hline \multirow{3}{*}{ Birds } & $30 \times 30$ & 0.060977 \\
& $100 \times 100$ & 0.003329 \\
& $300 \times 300$ & 0.00052 \\
Eiffel & $30 \times 30$ & 0.050091 \\
& $100 \times 100$ & 0.003736 \\
\multirow{4}{*}{ Stones } & $300 \times 300$ & 0.000065 \\
& $30 \times 30$ & 0.049013 \\
& $100 \times 100$ & 0.002993 \\
\hline \hline
\end{tabular}

Table 2 and Figure 9 show that there is an inverse relationship between the pixel colour value of number blocks and the correlation. This means that increasing the blocks size results in a lower correlation for all the cases as a result of using the scrambling technique. The process of dividing and shuffling the pixel colour value of of the image blocks will confuse the relationship between the original image and the scrambled image. Also, it minimises the relationship among image elements. Therefore, the perceivable information in the scrambled image is reduced by decreasing the correlation among the image pixels using the scrambling technique. 


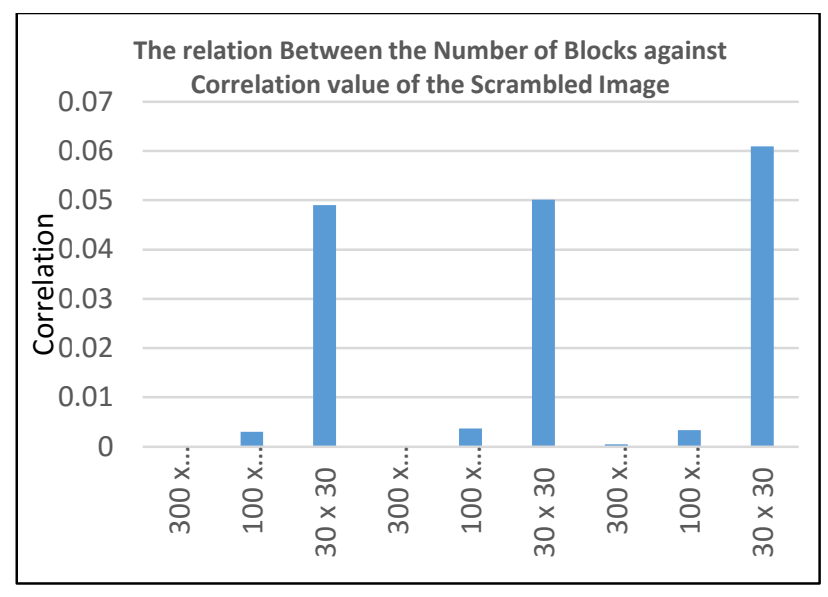

Fig. 9: different blocks size against correlation value of the scrambled image

\subsubsection{Analysis of the relationship between the iteration level and the correlation of the scrambled image for the birds image}

Four different iteration levels and three different block sizes were implemented for the scrambled image. The correlation obtained is tabulated in Table 3. This image has 40 levels. The other images are extracted using different iteration levels 1,10 , 20 and 30 with different block sizes $1 \times 1$ pixel, $3 \times 3$ pixels and $10 \times 10$ pixels.

Table 3: Correlation Results of the Scrambled Image (Birds) using Different Iteration Levels

\begin{tabular}{cccc}
\hline \hline Level & $(1 \times 1$ pixel $)$ & $(3 \times 3$ pixels $)$ & $(10 \times 10$ pixels $)$ \\
\hline Level 1 & 0.002125 & 0.028778 & 0.130654 \\
Level 10 & 0.000673 & 0.017675 & 0.099844 \\
Level 20 & 0.0003868 & 0.009834 & 0.074353 \\
Level 30 & 0.000298 & 0.005143 & 0.059834 \\
Level 40 & 0.0001853 & 0.002674 & 0.0465765 \\
\hline \hline
\end{tabular}

Table 3 and Figure 10 show that there is an inverse relationship between the iteration level and the correlation of the scrambled image for all cases. This means that by increasing the iteration level, a lower correlation for all the cases can be obtained by using the scrambling techniques.

The process of scrambling the pixel colour value of the image blocks by increasing the iteration level will increase the probability of confusing the relationship between the original image and the scrambled image. Also, it minimises the relationship among the image elements, therefore, the perceivable information in the scrambled image is reduced by decreasing the correlation among the image pixels using the scrambling technique.

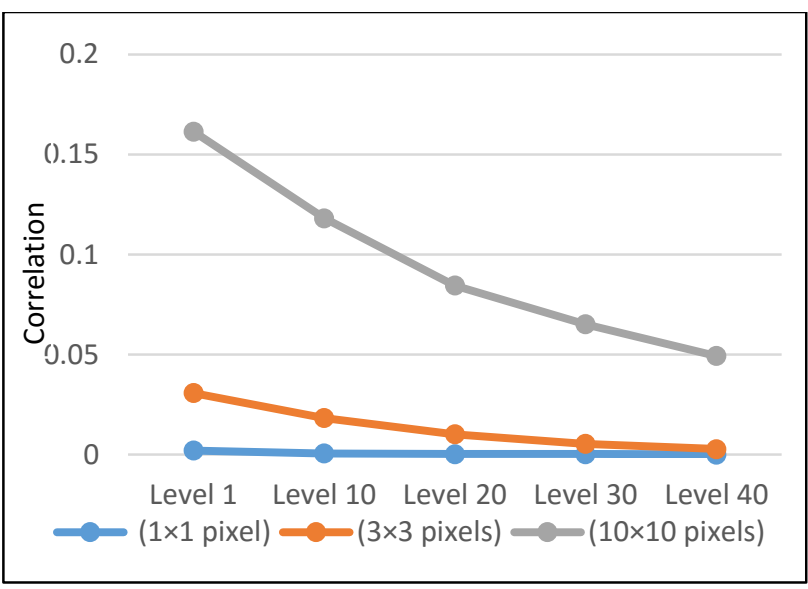

Fig. 10: Different Iteration Levels against Correlation of Scrambled Image (Birds)

\subsection{Information entropy}

Information theory is the mathematical theory of data communication and storage founded in 1949 by Shannon [25]. Information entropy is defined to express the degree of uncertainties in the system. It is well known that the entropy $H(m)$ of a message source $m$ can be calculated as:

$$
H(m)=\sum_{i=0}^{2 N-1} P(m) \log _{2} \frac{1}{P\left(m_{i}\right)}
$$

where $P\left(m_{i}\right)$ represents the probability of symbol $m_{i}$ and the entropy is expressed in bits. When the messages are encrypted or scrambled, their entropy should ideally be 8 . If the output of such a cipher emits symbols with an entropy of less than 8 , there exists a certain degree of predictability which can threaten the security of the image. In order to test and evaluate the effect of the pixel colour value of number blocks and the iteration levels of all the cases on the entropy value, a different number of block sizes and iteration levels have been used for these image cases.

\subsubsection{Analysis of the relationship between the Pixel} Colour Value of different blocks size and the entropy of the scrambled image

Table 4 and Figure 11 summarise the entropy value results of the scrambled image for all cases by using a different blocks size with different iteration levels.

Table 4: Entropy value results of the scrambled image for all cases

\begin{tabular}{ccc}
\hline \hline Case & $\begin{array}{c}\text { Number of } \\
\text { blocks }\end{array}$ & $\begin{array}{c}\text { Entropy of Scrambled } \\
\text { image }\end{array}$ \\
\hline \multirow{3}{*}{ Birds } & $30 \times 30$ & 7.736550 \\
& $100 \times 100$ & 7.758109 \\
& $300 \times 300$ & 7.785859 \\
Eiffel & $30 \times 30$ & 7.598373 \\
& $100 \times 100$ & 7.745343 \\
Stones & $300 \times 300$ & 7.767353 \\
& $30 \times 30$ & 7.695688 \\
& $100 \times 100$ & 7.758872 \\
\hline \hline
\end{tabular}




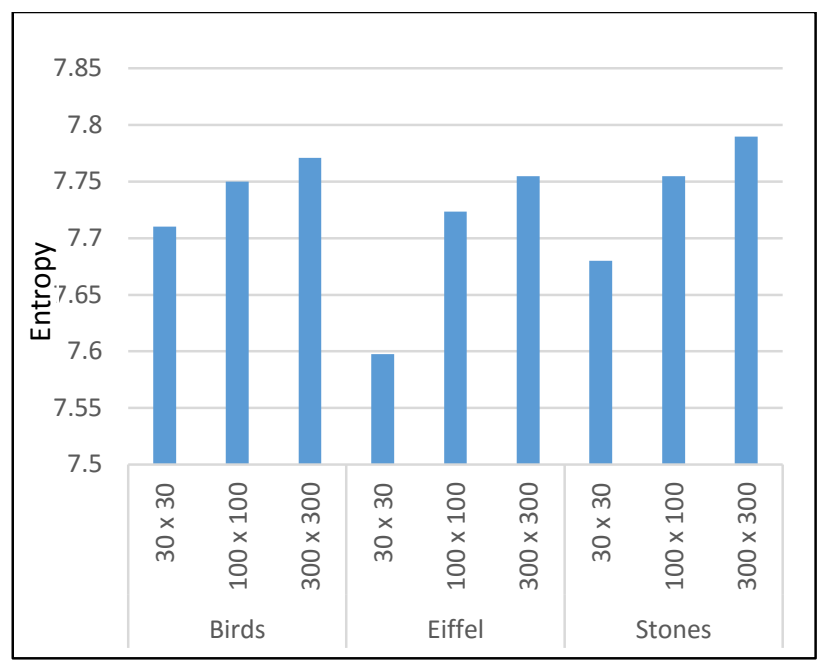

Fig. 11: Entropy value against pixel colour value of different blocks size of Scrambled image

Table 4 and Figure 11 indicate that there is a direct relationship between the pixel colour value of number blocks and the entropy value. this means that increasing the number of blocks that modified the pixel colour value results in a higher entropy value for all the cases by using the scrambling technique. The process of dividing and shuffling the pixel colour value of the image blocks will confuse the relationship between the original image and the scrambled image.

\subsubsection{Analysis of the relationship between the} iteration level and the entropy of scrambled image for the Eiffel image

Table 5 and Figure 12 illustrate the entropy results of the scrambled image for the Eiffel image against iteration level for all cases. Figure 12 illustrates the entropy values graphically. The objective of this test is to clarify the effect of iteration level on the entropy value for all cases.

Table 5: Entropy Results of the Scrambled Image (Eiffel) using different Iteration Levels

\begin{tabular}{cccc}
\hline \hline Level & $1 \times 1$ pixel & $3 \times 3$ pixels & $10 \times 10$ pixels \\
\hline Level 1 & 7.748436 & 7.716288 & 7.561724 \\
Level 10 & 7.754853 & 7.724342 & 7.570423 \\
Level 20 & 7.757965 & 7.726645 & 7.582087 \\
Level 30 & 7.75953 & 7.733538 & 7.59763 \\
Level 40 & 7.767353 & 7.745343 & 7.598373 \\
\hline \hline
\end{tabular}

Table 5 and Figure 12 indicate that the iteration level has an impact on the entropy value when using the scrambling technique. There is direct relationship between the entropy value and the iteration level for all cases of the scrambled images. This means that by increasing the iteration level, a higher entropy value for all the cases is obtained by using the scrambling techniques.

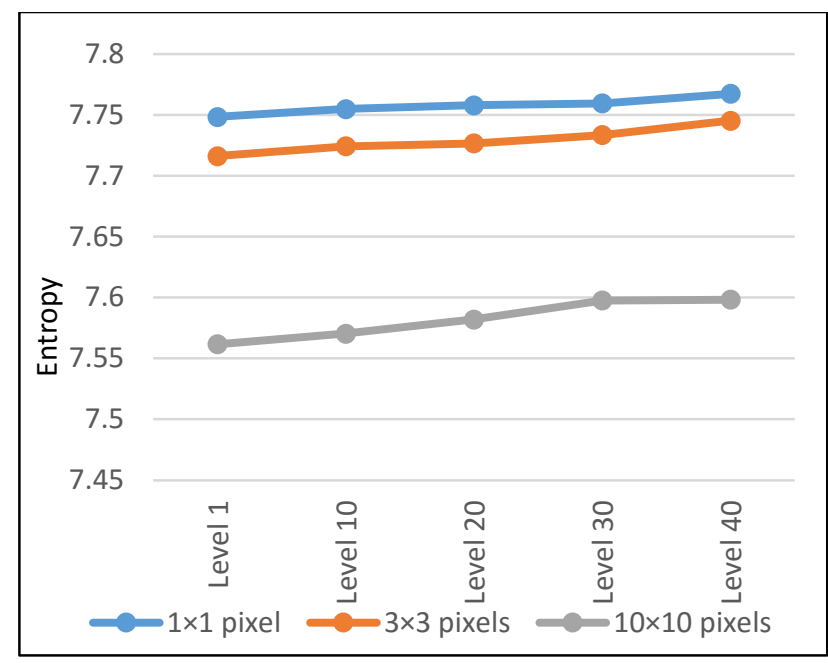

Fig. 12: Different Iteration Levels against Entropy of Scrambled Image (Eiffel).

\subsection{Image scrambling degree}

The scrambled image must have diffusion and confusion properties, which are the basis for designing practical ciphers. These two superior properties can be tested by computing the correlations of adjacent pixels in the scrambled image using the gray difference degree (GDD) function. To evaluate the effect of image scrambling, Ye and $\mathrm{Li}$ [26] introduced the gray difference and the gray degree of scrambling. The gray difference of a pixel with a neighbour pixel is defined as follows:

$G D(i, j)=\frac{1}{4} \sum_{i^{\prime}, j^{\prime}}\left[P(i, j)-P\left(i^{\prime}, j^{\prime}\right)\right]^{2}$

where $\left\{\left(i^{\prime}, j^{\prime}\right)=(i-1, j),(i+1, j),(i, j-1),(i, j+1)\right\}$ and $\mathrm{P}(\mathrm{i}, \mathrm{j})$ denotes the grey value at position $(\mathrm{i}, \mathrm{j})$.

After computing the gray difference for every pixel in the image, except the pixels at the edges, the whole average neighbourhood gray difference of the image can be computed by summing and averaging:

$E(G D(i, j))=\frac{\sum_{i=2}^{M-1} \sum_{j=2}^{N-1} G D(i, j)}{(M-2) \times(N-2)}$

The gray value degree is defined by

$G D D=\frac{\mathrm{E}^{\prime}(\mathrm{GD}(\mathrm{i}, \mathrm{j}))-\mathrm{E}(\mathrm{GD}(\mathrm{i}, \mathrm{j}))}{\mathrm{E}^{\prime}(\mathrm{GD}(\mathrm{i}, \mathrm{j}))+\mathrm{E}(\mathrm{GD}(\mathrm{i}, \mathrm{j}))}$

where $E(G D(i, j))$ and $E^{\prime}(G D(i, j))$ denote the average neighbourhood gray differences of the input and the scrambled images, respectively. The GDD value will be a number between -1 and 1 . Better scrambling corresponds to an absolute value near $1[20]$.

\subsubsection{Analysis of the relationship between the scrambled image and the scrambling techniques by using the scrambling degree}

Table 6 and Figure 13 summarise the scrambling degree results of the difference between the original image and the scrambled image for all cases by using a different pixel colour value of different blocks size with 40 iteration levels. 
Table 6: The Scrambling Degree Results of the Scrambled Image

\begin{tabular}{ccc}
\hline \hline Case & Number of blocks & Scrambling Degree \\
\hline \multirow{3}{*}{ Birds } & $30 \times 30$ & 0.8725 \\
& $100 \times 100$ & 0.9048 \\
Eiffel & $300 \times 300$ & 0.9396 \\
& $30 \times 30$ & 0.8822 \\
& $100 \times 100$ & 0.9193 \\
Stones & $300 \times 300$ & 0.9496 \\
& $30 \times 30$ & 0.9033 \\
& $300 \times 300$ & 0.926 \\
\hline \hline
\end{tabular}

Table 6 and Figure 13 indicate that the statistical results of the scrambling techniques have a high scrambling degree. The scrambling degree in all the cases is near 1 . That means there is a high relationship between the scrambling technique and the scrambled image. Hence the efficiency of the scrambling techniques is high. This indicates that there is a direct relationship between the number blocks and the scrambling degree. Increasing the number of blocks that modified the pixel colour value resulted in a higher scrambling degree for all the cases by using the scrambling technique, thus showing that the security of the developed scrambling methods is high. The process of dividing and shuffling the pixel colour value of the image blocks will confuse the relationship between the original image and the scrambled image.

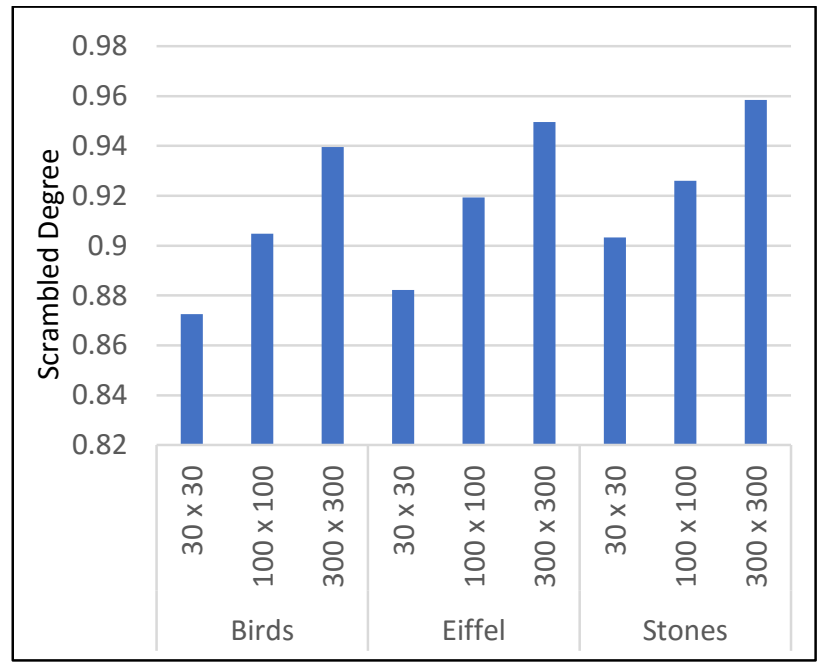

Fig. 13: The Scrambling Degree Results of the scrambled Image

\subsubsection{Analysis of the relationship between the iteration level and the scrambled images by using the scrambling degree (stones image)}

Table 7 and Figure 14 summarise the scrambling degree results between the Iteration Level and the scrambled image for all cases by using a different pixel colour value of number blocks and different iteration levels. The objective of this test is to clarify the effect of the iteration level on the scrambled images.
Table 7: Scrambling Degree Results of the Scrambled Image using Different Iteration Levels

\begin{tabular}{cccc}
\hline \hline Level & $(1 \times 1$ pixel $)$ & $(3 \times 3$ pixels $)$ & $\begin{array}{c}(10 \times 10 \\
\text { pixels })\end{array}$ \\
\hline Level 1 & 0.9049 & 0.8856 & 0.8576 \\
Level 10 & 0.9192 & 0.8992 & 0.874 \\
Level 20 & 0.9296 & 0.9104 & 0.8875 \\
Level 30 & 0.9408 & 0.9159 & 0.8934 \\
Level 40 & 0.9584 & 0.926 & 0.9033 \\
\hline \hline
\end{tabular}

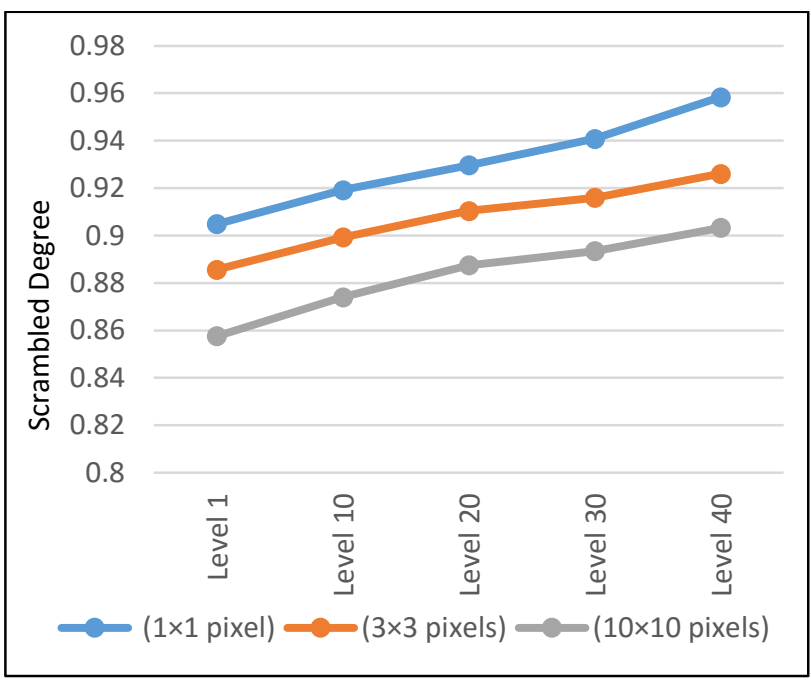

Fig.14: Iteration Level against Scrambling Degree of Scrambled Image (Stones image)

Table 7 and Figure 14 show that a direct relationship exists between the iteration level and the scrambling degree value of the scrambled image for all the cases, as the iteration level increases, the scrambling degree increases. This means that a higher scrambling degree results from using the developed techniques.

\subsection{Histogram analysis}

The histogram test illustrates how pixels of the scrambled images are distributed at each colour intensity level. An image histogram can be used to measure the statistical similarity between the original image and the scrambled image. Histograms illustrate how pixels in an image are distributed by plotting the number of pixels at each intensity level. Histograms are drawn for all the blocks for different block sizes. It is evident from the results obtained that the block/region level histograms of the scrambled image compared to the original image are reasonably uniform and evenly spread across all possible intensity levels in the original image. Figure 15 and Figure 16 illustrate the histogram of the original images and the histogram of the scrambled image graphically. 


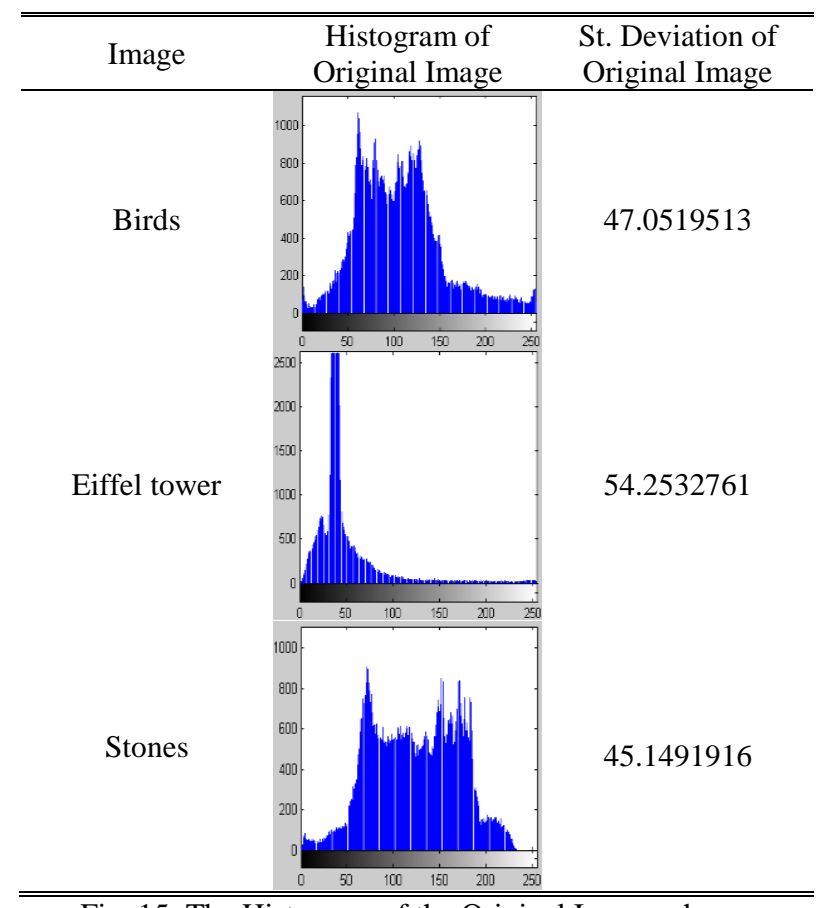

Fig. 15: The Histogram of the Original Images shown graphically

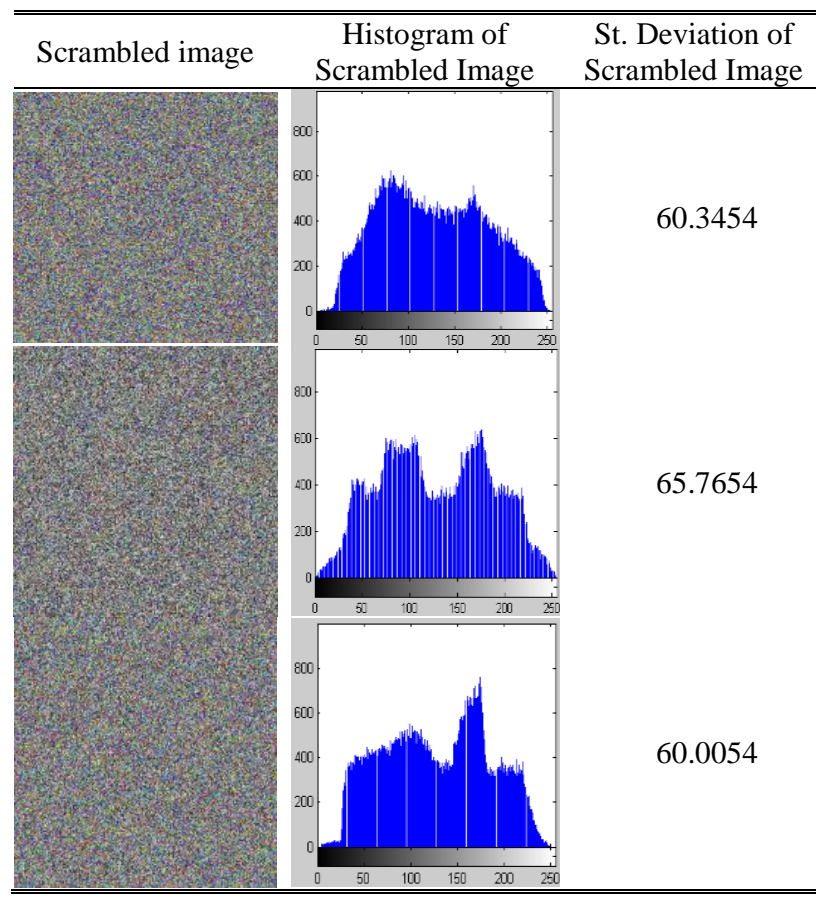

Fig. 16: The Histogram of the Scrambled Image shown graphically.

\section{DISCUSSION}

The scrambling technique has been implemented and tested to achieve the objectives of this paper. The correlation measure has been used to test and evaluate the impact of the number of blocks that modified the pixel colour value and the iteration level by using a scrambling technique. Experimental results of the scrambling technique show that an inverse relationship exists between the number of blocks that modified the pixel colour value and the correlation for all cases. It has also been illustrated that there is a direct relationship between the number blocks, the iteration level and the entropy value. This means that increasing the number of blocks that modified the pixel colour value and the iteration level results in a higher entropy value for all the cases. Furthermore, a direct relationship exists the number of blocks that modified the pixel colour value, the iteration level and the standard deviation value of the scrambled image for all the cases. As the number of blocks that modified the pixel colour value and the iteration level increases, the standard deviation increases. This means that a higher standard deviation is obtained by using the developed techniques. By amending the pixel colour values the statistical results show that the scrambling techniques have a high scrambling degree which in all the cases is near to 1 . That means there is a high relationship between the scrambling technique and the scrambled image. Hence the efficiency of the scrambling techniques is high. That illustrates the security of the developed scramble methods is also high.

As a result, the process of dividing and shuffling the pixel colour values of the image blocks confuses the relationship between the original image and the scrambled image. Moreover, the perceivable information in the scrambled image has been reduced by decreasing the correlation among the image elements. Furthermore, the process of dividing and modified the pixel clolour value of image blocks decreases the mutual information among the scrambled image variables. As a consequence, the entropy value is increased, the standard deviation is increased, the scrambling degree is high and the scrambled image histogram is different from the histogram of the original image.

\section{CONCLUSION}

A simple and strong technique has been developed for image security using a multilevel block and pixel based scrambling technique. The scrambling processes are used to divide the original image into a number of blocks that modified the pixel colour value (for example 3 pixels by 3 pixels) that are then scrambled. the pixel colour values in the image between the range of the colour ( 0 to 255 ) by using a vertical and horizontal scrambling based on a scrambling table. The security measurements of the original images have highly correlated elements. This means there is a good relationship between the elements of the original images, which also have a low entropy value, a large standard deviation and high scrambling degree. The correlation between the image elements is significantly decreased and the entropy value is significantly increased by using the developed techniques which show that an inverse relationship exists between the the number of blocks that modified the pixel colour value, iteration levels and correlation, while there is a direct relationship between the number of blocks that modified the pixel colour value, iteration levels and the entropy as well as the scrambling degree. The developed techniques are expected to show good performance, low correlation and high entropy, the standard deviation increases or decreases and the high scrambling degree increases and the scrambled image histogram is different from the histogram of the original image. 


\section{REFERENCES}

[1] Sekgwathe, Virginiah Talib, and Mohammad. Cyber Crime Detection and Protection: Third World Still to Cope-Up. in e-Technologies and Networks for Development. 2011. Tanzania: Springer.

[2] Lin, K.T., Information hiding based on binary encoding methods and pixel scrambling techniques. Applied optics, 2010. 49(2): p. 220-228.

[3] Lestriandoko, N.H. and T. Wirahman. Reversible watermarking using difference of virtual border for digital image protection. in International Conference Distributed Framework and Applications (DFmA). 2010. Yogyakarta: IEEE.

[4] van Renesse, R.L. Hidden and scrambled images: A review. in Proceedings of the SPIE Electronic Imaging. 2002. Santa Clara, CA: International Society for Optics and Photonics.

[5] Huang, H.-f. An Image Scrambling Encryption Algorithm Combined Arnold and Chaotic Transform. in International Conference of China Communication (ICCC 2010 E-BOOK). 2010. China: Scientific Research Publishing.

[6] Fang, L. and W. YuKai. Restoring of the watermarking image in Arnold scrambling. in Signal Processing Systems (ICSPS), 2nd International Conference. . 2010. Dalian: IEEE.

[7] Liu, Z., et al., Image encryption by using gyrator transform and Arnold transform. Journal of Electronic Imaging, 2011. 20(1): p. 13 - 20.

[8] Abuturab, M.R., Securing color information using Arnold transform in gyrator transform domain. Optics and Lasers in Engineering, 2012. 50(5): p. 772 - 779.

[9] Wu, L., et al. Arnold Transformation Algorithm and Anti-Arnold Transformation Algorithm. in 1st International Conference Information Science and Engineering (ICISE). 2009. Nanjing: IEEE.

[10] Zhou, Y., et al., Image encryption using P-Fibonacci transform and decomposition. Optics Communications, 2012. 285(5): p. 594-608.

[11] Wang, Y.-Y., et al., Optical image encryption based on binary Fourier transform computer-generated hologram and pixel scrambling technology. Optics and Lasers in Engineering, 2007. 45(7): p. 761-765.

[12] Ye, G., Image scrambling encryption algorithm of pixel bit based on chaos map. Pattern Recognition Letters, 2010. 31(5): p. 347-354.

[13] Wang, Y., et al., A new chaos-based fast image encryption algorithm. Applied soft computing, 2011. 11(1): p. 514-522.

[14] Ye, R., A novel chaos-based image encryption scheme with an efficient permutation-diffusion mechanism. Optics Communications, 2011. 284(22): p. 5290-5298.

[15] Wang, X., L. Teng, and X. Qin, A novel colour image encryption algorithm based on chaos. Signal Processing, 2012. 92(4): p. 1101-1108.

[16] Zhou, Y., K. Panetta, and S. Agaian. An image scrambling algorithm using parameter bases Msequences. in International Conference on Machine Learning and Cybernetics. 2008. Kunming: IEEE.

[17] El-Latif, A.A.A., X. Niu, and N. Wang, Chaotic image encryption using bézier curve in DCT domain scrambling, in Digital Enterprise and Information Systems. 2011, Springer. p. 30-41.
[18] Gu, G.-S. and G.-Q. Han. The application of chaos and DWT in image scrambling. in Machine Learning and Cybernetics, 2006 International Conference on. 2006: IEEE.

[19] Premaratne, P. and M. Premaratne, Key-based scrambling for secure image communication, in Emerging Intelligent Computing Technology and Applications. 2012, Springer: Huangshan, China. p. 259-263.

[20] Qadir, F., M. Peer, and K. Khan, Digital Image Scrambling Based on Two Dimensional Cellular Automata. International Journal of Computer Network and Information Security (IJCNIS), 2013. 5(2): p. 3641.

[21] Liehuang, Z., et al., A novel image scrambling algorithm for digital watermarking based on chaotic sequences. International Journal of Computer Science and Network Security, 2006. 6(8B): p. 125-130.

[22] Abugharsa, A.B., A.S.B. Hasan Basari, and H. Almangush, A New Image Scrambling Approach using Block-Based on Shifted Algorithm. Australian Journal of Basic \& Applied Sciences, 2013. 7(7): p. 570-579,

[23] Burger, W. and M. Burge, Digital image processing: an algorithmic introduction using Java. 2008: Springer-Verlag New York Inc.

[24] El-din., H., et al., Encryption quality analysis of the RC5 block cipher algorithm for digital images. Optical Engineering, 2006. 45( 10): p. 102 - 111.

[25] Shannon, C.E., Communication theory of secrecy systems. Bell system technical journal, 1949. 28(4): p. 656-715.

[26] Ye, R. and H. Li. A novel image scrambling and watermarking scheme based on cellular automata. in Electronic Commerce and Security, 2008 International Symposium on. 2008: IEEE.

[27] J. M. Guo, H. Prasetyo, H. Lee, C. C. Yao, "Image retrieval using indexed histogram of void-and-cluster block truncation coding", Signal Process., Jan 2016, vol. 123, pp. 143-156.

[28] HM Almangush, MKA Ghani, AB Abugharsa., "Multilayer Reversible Data Hiding Based on Histogram Shifting with High Quality and Capacity", International Review on Computers and Software (IRECOS)., 2015 , vol. 8, No. 10 . 